Nach einer sorgfältigen Behandlung mit Sublimatalcohol kann man endlich eine ganze Reihe von Thieren trocken conserviren, wenn man hierbei einige Kunstgriffe anwendet. Das erste Erforderniss hierzu ist ein langes, womöglich mehrwöchiges Verweilen derselben in dieser Flüssigkeit. Werden sie dann herausgenommen, so lasse man sie nicht schnell austrocknen, sondern bedecke sie, um dies zu verhindern, mit einem Kasten oder dergl. Gleichzeitig stopfe man durch die Körperöffnungen Watte und ähnliche Stoffe nach, um die Haut immer prall zu erhalten. Dies lässt sich mit Glïck allerdings nur bei Thieren mit fester, harter Körperbedeckung ausführen, wie bei Schlangen, Eidechsen und einer Anzahl von Fischen. Andere Objekte, wie Frösche kann man aufblasen, oder besser noch, mit trocknem Sande ausfüllen, den man nach dem Trocknen wieder ausschïttet. Ein sehr geeignetes Füllmaterial geben ferner Flüssigkeiten ab, die später erhärten, wie etwa eine vergiftete Leimabkochung, Paraffin, Fett etc., die man mittels einer Spritze in die Leibeshöhle einführt. Wo Gliedmaassen vorhanden sind, wie bei Säugern. Fröschen etc. ist es gut, an passender Stelle eine Oeffnung herzustellen, um von dort aus das Material einzuführen.

Ein derartiges Präparationsverfahren eignet sich namentlich für kleinere Thiere, deren Abbalgen nicht ganz leicht und deren Ausstopfen schwierig ist, wie kleine Mäuse, Salamander, Eidechsen, Blindschleichen etc. Die so hergestellten Präparate haben weniger einen wissenschaftlichen, als vielmehr einen pädagogischen Zweck und können den Anschauungsunterricht mehr fördern, als in Gläsern und Alcohol eingeschlossene, und bei geschickter Behandlung bewahren sie ihre natürlichen Formen besser, als ausgestopfte Bälge, die zumeist etwas Gekünsteltes an sich haben.

\title{
A. B. Meyer, Abbildungen von Vogel-Skeletten,
}

herausgegeben mit Unterstütung der Generaldirection der K. Sammlungen für Kunst und Wissenschaft in Dresden. Band I. Mit $121 \mathrm{~T}$ afeln (I-CXX) in Lichtdruck. Dresden 1879-88.

\section{Bericht von M. Fürbringer.}

Die 12. und 13. Lieferung von A. B. Meyer's Abbildungen von Vogelskeletten ist am Anfange dieses Jahres*) edirt worden.

*) Das Manuscript wurde 1890 eingesandt. 
Mit dieser 12. Lieferung hat der erste Band dieses hervorragenden Werkes, dessen erste Lieferung im Jahre 1879 veröffentlicht wurde, seinen Abschluss erreicht und zugleich verbürgt die damit herausgegebene 13. Lieferung, die erste des zweiten Bandes, die weitere Fortführung dieses in hohem Grade verdienstvollen und dankenswerthen Unternehmens.

Oft genug ist in ornithologischen und zoologischen Zeitschriften und Jahresberichten beim Erscheinen der einzelnen Lieferungen des Meyer'schen Werkes gedacht worden; sei es auch mir jetzt gestattet, mit kurzen Worten auf den Inhalt und die Bedeutung dieser Veröffentlichung des um die Kenntniss des indoaustralischen Archipels, um die Ornithologie, Anthropologie und die zoologische Wissenschaft im Allgemeinen wie um die Museumskunde hochverdienten Forschers und Gelehrten hinzuweisen.

Der vorliegende erste Band enthält auf 71 Seiten Text in Grossquart und 120 in der renommirten Kunstanstalt von Wilhelm Hoffmann angefertigten Lichtdrucktafeln von demselben Formate die Beschreibungen und Abbildungen von 116 Vogelspecies nebst 27 Rassevögeln, welche sich nach dem vom Verfasser gegebenen Inhaltsverzeichnisse auf 47 Familien vertheilen. In ausgedehnter Weise sind hierbei vertreten die Psittaci (Stringopidae, Cacatuidae Psittacidae und Trichoglossidae), und zwar mit 23 Species, die Passeres (Cotingidae, Muscicapidae, Dicruridae, Oriolidae, Laniidae, Meliphagidae, Pycnonotidae, Tanagridae, Sturnidae, Corvidae und Paradiseidae) mit 27 Species (worunter 7 Paradiesvögel), die Columbidae (Columbidae und Didinculidae) mit 9 Species und 19 Rassetauben, die Galli (Phasianidae und Tetraonidae) mit 10 Species und 8 Rassehühnern, die Rallidae mit 8 Species. Je 4 Arten kommen auf die Alcedinidae und Anatidae, 3 auf die Strigidae, je 2 auf die Bucerotidae, Meropidae, Trochilidae, Charadriidae (Charadriidae und Scolopacidae), Alcidae (Urïdae und Alcidae), Ardeidae, Pelargi (Ciconidae und Plataleidae) und Pelecanidae, je 1 auf die Rhamphastidae, Cuculidae, Coliidae, Coraciidae, Cypselidae, Menuridae*), Gruidae, Psophiidae, Cariamidae, Otididae, Laridae, Procellariidae und Apterygidae. Darunter befinden sich zwei A. B. Meyer'sche Species (Xanthotis rubiensis und Notornis Hochstetteri), sowie zahl-

*) Die von den meisten Autoren den Passeres eingereihten Menuridae trenne ich auf Grund meiner Untersuchungen nebst den Atrichiidae von den Passeres ab und stelle sie zwischen diese und die Pici im weiteren Sinne. 
reiche für die Vogelanatomie in höherem Grade interessante · Gattungen, wie Stringops, Nestor, Menura, Chasmorhynchus, Heteralocha, Manucodia, Otidiphaps, Didunculus, Ocydromus, Notornis, Psophia, Cariama, Apteryx u. a. Ein nicht geringer Theil der abgebildeten Skelette wurde von dem Verfasser selbst auf seinen Reisen erbeutet und befindet sich nebst den anderen abgebildeten Stücken in dem unter seiner Direction stehenden Königlichen Zoologischen Museum zu Dresden.

Die Abbildungen enthalten 134 seitliche Gesammtansichten von Skeletten; der eine dem Beschauer zugekehrte Flïgel ist, um alle seine Theile ohne Verkürzung sehen zu lassen, senkrecht nach oben luxirt und zeigt somit die Ventralseite, der andere wurde in der Regel vom Skelette abgelöst und in einer dem ersten Flïgel entsprechenden, somit die Dorsalfläche darbietenden Lage neben demselben abgebildet; von den Beinen befindet sich das eine von der lateralen Seite gesehene in natürlicher Lage, während das andere ebenfalls abgelöst und neben dem Skelette in ganzer Länge oder wenigstens in seinem distalen Abschnitte von der Medialseite zu beobachten ist. Bei vielen Skeletten ist an der einen unteren Extremität die natürliche Beinbekleidung erhalten. Ein Skelet, das von Brotogerys tirica, zeigt sämmtliche Knochen exarticulirt. $\mathrm{Zu}$ diesen Skeletansichten treten bei vielen Species, insbesondere auch bei den Rassetauben, separate Ansichten des Schädels von oben, von unten und von der Seite; ebenso sind einige Medianschnitte und Ventralansichten (Gaumenansichten), sowie Ansichten von hinten und schräg von vorn und von der Seite gegeben; Einzelabbildungen des Unterkiefers finden sich wiederholt. Besonders bevorzugt wurden die Schädel der Tauben, sowie diejenigen von Balearica pavonia und von Notornis Hochstetteri Letztere Species enthält auch ausser der Gesammitansicht des ganzen Skelettes auf weiteren drei Tafeln das osteologische Detail der einzelnen Skelettheile in natürlicher Grösse. Fernere Tafeln und Figuren dienen der Darstellung des Zungenbeins, der trachealen Windungen bei den Species von Manucodia, der Syrinxpauke von Anas boschas und des Scleralringes bei den Eulen.

Der Text enthält anf 15 einleitenden Seiten (p. I-XV) ausser Titel und Widmung ein systematisches Verzeichniss und einen alphabetischen Index, wodurch die schnellstmögliche Orientirung: in dem Inhalte dieses ersten Bandes gesichert wird, Berichtigungen und Zusätze zu Band I und die Liste der Werke, deren anato- 
mische Nomenclatur hauptsächlich berücksichtigt wurde. Daran schliesst sich der eigentliche Text mit den Beschreibungen und Maassen der abgebildeten Skelette an (p. 1-71). Diese Besprechungen enthalten Literaturnachweise, Bemerkungen über Herkunft, Aufbewahrungsort mit Katalognummer und Erhaltungszustand *) der Skelette, sowie anatomische Bemerkungen, in denen die wichtigeren Besonderheiten hervorgehoben, auch hier und da Vergleichungen mit den Verwandten und entwickelungsgeschichtliche Excurse gegeben werden; bei den selteneren und interessanteren Arten, insbesondere bei Notornis Hochstetteri, sind diese Beschreibungen in grösster und erwünschtester Ausführlichkeit gehalten. Die bei jedem Skelette angegebenen Maasse (in Millimetern) betreffen Länge und grösste Breite des Cranium, Länge des Humerus, der Ulna, des Radius, der Manus, des Femur, der Tibia und des Tarso-Metatarsus, sowie Länge und grösste Breite des Brustbeines (nebst Höhe der Crista sterni) und des Beckens; auch hier sind die selteneren Species, so namentlich Notornis Hochstetteri durch zahlreichere Messungen (ausser den allgemein durchgeführten Maassen auch die proximale und distale Breite des Humerus, der Ulna, des Femur, der Tibia und des Tarso-Metatarsus, die Länge einzelner Zehen, die Maasse des Coracoid, der Furcula und der Scapula, die Breite des Sacrum etc.) ausgezeichnet. -

Mehr oder minder ausführliche bildliche Darstellungen des Skeletsystems der Vögel besitzen wir bereits seit langer Zeit. Ganz abgesehen von den zahlreichen, z. Th. ausgezeichnet illustrirten osteologischen Monographien von Pander und d'Alton, Yarrell, Burmeister, Owen, P. Gervais, Huxley, W. K. Parker, Murie, M. Schmidt, Garrod, Forbes, Watson, Filhol, Marsh, Shufeldt u. v. A., sei insbesondere an Eyton's Osteologia avium und die grossen Prachtwerke von A. Milne-Edwards und Grandidier erinnert; auch sind, namentlich nach dem Erscheinen der ersten Lieferungen von Meyer's Abbildungen von Vogelskeletten und wohl z. Th. durch die hier gebotene Art der Darstellung angeregt, wiederholt photographische Reproductionen einzelner Skelette oder Theile des Skeletsystems gegeben worden. Ein in so grossem Maassstabe unternommenes und so consequent durchgeführtes Unternehmen wie das Meyer'sche, das uns in dem vorliegenden

*) Die wenigen Defecte oder pathologischen Veränderungen der abgebildeten Skelette sind mit der grössten Gewissenhaftigkeit hervorgehoben. 
ersten Bande bereits mit phototypischen Skelettdarstellungen nebst Beschreibungen und Messungen von 145 Species und Rassen beschenkt, findet sich jedoch meines Wissens nirgends wieder. Eine wie grosse und von mir voll anerkannte Bedeutung die angeführten osteologischen Werke neben ihrem hohen ästhetischen Werthe auch besitzen, an wissenschaftlicher Brauchbarkeit für den Untersucher werden sie von dem hier besprochenen übertroffen. Mit seinen absolut getreuen und wahrhaftigen, weil der Phantasie des reproducirenden Künstlers nicht überlassenen, sondern lediglich durch physikalisch-chemische Processe nach der Natur copirten Abbildungen bildet dasselbe eine über jeden Zweifel sichere und zuverlässige Grundlage für jede wissenschaftliche Untersuchung über das Skelettsystem der Vögel. Dazu kommen die zahlreichen vom Verfasser ausgeführten Messungen, von deren Richtigkeit sich der Referent zu wiederholten Malen überzeugen konnte, ferner die bei aller Kürze von kundiger Hand zeugenden osteologischen Notizen und die bedeutsamen Hinweise auf Entwickelung, Resultate der Züchtung etc.

Ueber den Werth genauer osteologischer Messungen zum Zwecke systematischer Ergebnisse braucht jetzt nicht mehr discutirt zu werden; jeder genaue Arbeiter auf diesen Gebieten hat sich davon überzeugt und er lernt zugleich mit jeder neuen Untersuchung das Wechselnde und Constante, die individuellen Variirungen und die durchgreifenden, typischen Merkmale besser und genauer unterscheiden. Solche Untersuchungen können, bei dem innerbalb der Art obschon meist in bescheidenen Grenzen wechselnden Verhalten, nicht oft genug wiederholt werden und jeder neue Beitrag, der neues Material zur Durcharbeitung und Vergleichung herbeibringt, ist dankbar zu begrïssen.

Es wäre aber viel zu wenig gesagt, wollten wir Meyer's Abbildungen von Vogelskeletten nur als einen Beitrag in diesem Sinne bezeichnen. Hier liegt vielmehr ein Werk von ganz hervorragender und in gewissem Sinne unvergleichlicher Bedeutung vor, welches geeignet ist, denjenigen, die nicht das Glück haben über grosse Sammlungen von Skeletten zu verfügen, Ersatz dafür zu bieten, - und zugleich ein Monumentum aere perennius, das, wie auch im Laufe der Zeiten die Anschauungen und Deutungen wechseln mögen, immer bestehen und niemals veralten wird.

In der Natur eines solchen, auf mehrere Bände berechneten Werkes liegt von selbst, dass der eine Band auf diese, der andere 
auf jene Familien und Ordnungen den Schwerpunkt legt; das Streben nach ganz gleichmässiger Vertheilung würde zur Verflachung führen. So sind auch in diesem ersten Bande gewisse Familien mit Vorliebe berïcksichtigt und man darf die Auswahl dieser Familien und ihrer Repräsentanten als eine sehr glïckliche bezeichnen. Das lässt auch für die Auslese in den folgenden Bänden eine durchaus günstige Prognose stellen: der Verfasser beherrscht das von ihm behandelte Gebiet materiell und formell vollkommen und weiss seinen Stoff in meisterhafter Weise zu vertheilen, er verfügt dabei über die reichen Schätze eines grossen Museums und er hat ausserdem durch seine Veröffentlichungen eine Vertrauensstellung gewonnen, die ihm auch in anderem Besitze befindliche ornithologische Seltenheiten ersten Ranges zugänglich und für sein Werk brauchbar machen wird.

Auch der Aufstellung und sonstigen Anordnung der abgebildeten Gesammtskelette und Skelettheile ist nur zuzustimmen und es kann kaum eine andere Darstellung gedachi werden, die sich besser als diese Phototypien eignete, um nach ihnen zuverlässige eigene Messungen auszuführen, An die getreue Benutzung des Meyer'schen Werkes wird sich eine Vertiefung der osteologischen Methode in der Ornithologie anschliessen.

Mehrfache Tafeln behandeln, wie schon oben bemerkt, das osteologische Detail; hier seien besonders die schönen und übersichtlichen Schädelansichten der Rassetauben, sowie die Notornis betreffenden Abbildungen hervorgehoben. Nach diesen Anfängen kann man auch für die weiteren Tafeln grosse Erwartungen hegen und der Verfasser wird wohl Vieler Wünsche erfüllen, wenn er diese Schädelansichten (von oben, von unten, von der Seite und von hinten) auf alle wichtigeren Typen der Vogelwelt ausdehnt und damit zu detaillirten Schädeluntersuchungen und zu eigener Kenntnissnahme und Prüfung der von Huxley, W. K. Parker, Garrod, Magnus, Forbes, M. Walker, Heerwagen u. A. hervorgehobenen Schädelmerkmale Gelegenheit giebt. Aehnliches gilt für grössere Zusammenstellungen von ventralen Ansichten von Brustbein (mit und ohne Brustgürtel) und Becken, sowie für die Ausdehnung der Messungen auf die Wirbel, auf Scapula, Coracoid und Clavicula, sowie auf die einzelnen Elemente der Hand und des Fusses.

Möge der Verfasser in diesen zuletzt ausgesprochenen Hoffnungen und Wünschen lediglich den zu den grössten Erwartungen 
sich steigernden Ausdruck der hohen Werthschätzung seines Werkes erblicken. Jene Genugthuung, die als stete Frucht getreuer und weiterblickender Arbeit freudig den inneren Menschen erfüllt, trägt er in sich und er weiss, dass darin auch die einzig wahre, bleibende und über jeden äusseren Wechsel erhabene Belohnung des Forschers liegt. Möge er aber auch aus den hier gegebenen Ausführungen die Ueberzeugung gewinnen, dass sein Werk, dieses werthvolle Geschenk, das er dem ornithologischen Publikum gegeben, in den unveräusserlichen Besitz desselben übergehen und dort für alle Zukunft eine nie versiegende Fundgrube bilden wird.

Mit der Aeusserung des aufrichtigsten Dankes für das bereits Gebotene, mit den besten Wünschen für das noch zu Erwartende sei diese Besprechung geschlossen.

\section{Jugendkleider einiger Hühnerarten. \\ Von}

Professor Dr. Altum, in Eberswalde.

Seit etwa 35 Jahren hat sich in den Katalogen der Händler das Angebot von „Dunenkleidern" und darunter selbstverständlich auch unserer Wildhühner sehr gesteigert. In Privat- wie öffentlichen Sammlungen findet man nicht selten recht reichhaltige Collectionen dieser interessanten ersten Kleider. Allein die auf diese folgenden sehr wechselvollen und vielfach ineinander greifenden "Jugendkleider" (Jugend-Contourkleider) sind und bleiben in den Sammlungen rarae aves. Ich erinnere mich nicht, die sämmtlichen Jugendkleider irgend einer Hühnerart in ununterbrochener Reihefolge an einer und derselbsn Stelle gesehen zu haben, wie wir z. B. eine solche Reihe von 15 jungen Fasanen jetzt besitzen. Wer sich von solchen Jugendgefiederreihen unterrichten will, müsste schon in vielen Sammlungen Umschau halten. Ich verkenne übrigens die Schwierigkeit einer solchen um die Förderung der Kenntniss unserer einheimischen Vogelwelt ohne Zweifel sehr verdienstvollen Arbeit keineswegs. Möchten die folgenden Beschreibungen einiger Jugendkleider unserer akademischen Sammlung zur Fortsetzung seitens Anderer, denen ein reichlicheres Material zur Verfügung steht, anregen! 


\section{$2 \mathrm{BHL}$ Biodiversity Heritage Library}

1891. "A. B. Meyer, Abbildungen von Vogel-Skeletten." Journal fu

r Ornithologie 39, 86-92. https://doi.org/10.1007/bf02252223.

View This Item Online: https://www.biodiversitylibrary.org/item/101710

DOI: https://doi.org/10.1007/bf02252223

Permalink: https://www.biodiversitylibrary.org/partpdf/142944

\section{Holding Institution}

Smithsonian Libraries

\section{Sponsored by}

Biodiversity Heritage Library

\section{Copyright \& Reuse}

Copyright Status: Public domain. The BHL considers that this work is no longer under copyright protection.

This document was created from content at the Biodiversity Heritage Library, the world's largest open access digital library for biodiversity literature and archives. Visit BHL at https://www.biodiversitylibrary.org. 